This is the final peer-reviewed accepted manuscript published in Regional Science and Urban Economics (Elsevier), Why not in your backyard? On the location and size of a public facility, Bellettini Giorgio, Kempf Hubert, 2013

The final published version is available online at:

https://doi.org/10.1016/i.regsciurbeco.2012.11.001

(C) [2013]. This manuscript version is made available under the Creative Commons AttributionNonCommercial-NoDerivs (CC BY-NC-ND) 4.0 International License (http://creativecommons.org/licenses/by-nc-nd/4.0/)

(This item was downloaded from IRIS Università di Bologna (https://cris.unibo.it)

When citing, please refer to the published version. 


\title{
Why not in your backyard? On the location and size of a public facility
}

\author{
Giorgio Bellettini ${ }^{\mathrm{a}, \mathrm{b}}$, Hubert Kempf ${ }^{\mathrm{c}, \mathrm{d}, *}$ \\ a University of Bologna, Italy \\ ${ }^{\mathrm{b}}$ CESifo, Germany \\ c Ecole Normale Supérieure de Cachan, France \\ d Paris School of Economics, France \\ * Corresponding author: hubert.kempf@univ-paris1.fr
}

\begin{abstract}
A B S T R A C T
In this paper, we tackle the issue of locating and sizing a public facility which provides a public good in a closed and populated territory and generates differentiated benefits to households. In the case of a "Nimby" ("Imby") facility, the smaller is the distance, the smaller (larger) is the individual benefit. Multiple solutions may arise in both cases, and we derive sufficient conditions for uniqueness in both cases. Optimal decisions on location and size are interrelated and depend on the complementarity or substitutability properties of size and distance in the utility function. Then we introduce a common-agency lobbying game, where agents attempt to influence the location and provision decisions by the government. Even when only a subset of households lobbies, the solution of the lobbying game can replicate the optimal solution. Under-provision or over-provision of the public good may be obtained both in the Nimby and the Imby cases. Finally, some non-lobbying households may be better off than in the case where all households lobby, which raises the possibility of free-riding at the lobbying stage.
\end{abstract}

JEL classification: R12 R53 558

Keywords: Facility location Nimby Lobbies

\section{Introduction}

The location of a public facility in a territory, providing a public good to the inhabitants of this territory, is a thorny issue. The main reason is that, in most cases, a public facility has differentiated effects on inhabitants, depending on distances. The importance of this issue can hardly be dismissed. The decision over the siting of such facilities is often a very complicated endeavor, especially in the case of obnoxious facilities, such as landfills, incinerators, prisons, etc. Although everybody acknowledges the importance of the public good, local residents usually oppose the construction of these facilities in their community, showing an attitude which is referred to as the "Nimby" ("Not In My Backyard") syndrome. The choice of the location of these facilities is the object of fierce political struggles, which lead to long and laborious decision processes and sometimes siting stalemates. ${ }^{1}$

We are very grateful to an anonymous referee and the Editor Yves Zenou for very useful comments. Earlier drafts of this paper were presented at the Public Economic Theory Conference in Beijing and seminars at University of Paris-I PantheonSorbonne, University of Bologna and University of Florence. Comments by participants are gratefully acknowledged. We are especially indebted to Stéphane Rossignol for comments and suggestions.

\footnotetext{
${ }^{1}$ Examples of public processes related to decisions on the siting of such facilities in Canada, the Netherlands and Switzerland can be found in Kuhn and Ballard (1998), Wolsink (1994) and Frey et al. (1996), respectively.
}

To analyze these issues, we set up a model of one locality, which we call "city", formed of households spread on a two-dimensional closed territory. A decision must be taken on the siting and the size of a facility, when households' utilities are affected both by the amount of the provision of the public good (i.e., the size of the facility) and the distance to the site of the facility. We refer to the facility as a "Nimby facility" when the relationship between individual benefits and distance is positive, and as an "Imby facility" when the relationship is negative. Thus, for a given amount of public good provision, the closer to a household is a facility, the worse it is for this household in the Nimby case and the better in the Imby case. ${ }^{2}$

First, addressing the joint issue of location and size from a normative point of view, we prove the existence and characterize the optimal solution that would be chosen by a benevolent social planner. More precisely, we find that the optimal decision on size and location depends on the relation between these two characteristics in the utility function. Two effects are at work here: a "congestion effect" such that shorter distance is associated with lower demand of the public good and an "agglomeration effect" such that shorter distance is associated with higher demand of the public good. ${ }^{3}$ In brief, the optimal decision

\footnotetext{
2 Ahlfeldt and Maennig (2010) provide a recent empirical analysis of a public facility the benefits of which vary with distance.

3 This effect is reminiscent of the agglomeration effect used in economic geography, as it refers to a positive effect due to increased density. Here it refers to a public good, and not to the concentration of production factors. On an attempt to combine both aspects, see Thisse and Wildasin (1992).
} 
on size and location depends both on the type of the facility, whether it is Nimby or Imby, and on the relationship between the public good and distance. Moreover we prove that, in either case, the optimal location may not be unique unless additional conditions are introduced, which depend on concavity and, in the case of Nimby facilities, on the curvature of the utility function. Lastly, it may be that the optimal provision of the public good is null when it is Nimby: the facility is not operating. This comes from the fact that, if operating, it would be too close to households and create too much harm due to distance.

Next, we turn to a positive political economy perspective to investigate how a jurisdiction would solve this two-dimensional problem and how the political solution can be compared to the optimal one which has previously been defined. Building on the menu-auction framework developed by Bernheim and Whinston (1986) and applied by Grossman and Helpman in a series of studies (Grossman and Helpman, 1994; Grossman and Helpman, 2001), we focus on the equilibrium of the lobbying game played by the government and the households, using as a benchmark the social planner solution. ${ }^{4}$

We show that the lobbying equilibrium replicates the optimal solution not only when all households lobby, but also when only some households lobby. With respect to the issue of the provision of the public good, we prove that, both in the Nimby and the Imby cases, when the lobbying equilibrium differs from the social planner solution, there may be either under-provision or over-provision of the public good.

Exploring further the issue of the consequences of the lobbying activities, we show that, when the equilibrium decision differs from the optimal one, some lobbies may be worse off whereas some non-lobbies may be better off. Interestingly, this result does not depend on a symmetry assumption where, by chance, two lobbies have exactly opposite preferences and therefore cancel each other's influences. This raises the issue of free-riding on lobbying activities. Therefore our analysis of the political decision of locating a public facility sheds new light on the distributional consequences of this problem.

A recent study by Feinerman et al. (2004) is close in spirit to ours, as it focuses on the political game being played by lobbies about the location of a waste facility. However it differs from ours in many ways. First, their model is very different, as it is based on a housing price mechanism in a two-city economy, where the two cities are situated at the extremities of a segment. The siting of the facility has only indirect effects on households' utility through the housing price, but does not enter directly in their utility function: as such, it does not properly match the definition of a Nimby facility, that is, an overall advantageous good generating local nuisances based on distance. Hence, they cannot address the link between the location and the supply of the public good. Second, our analysis is more general as our formalization allows us to study the location and size of a public facility having differentiated effects on an indefinite number of inhabitants disseminated in a territory, be they positive or negative. In other words, our analysis is not restricted to obnoxious facilities, nor to a one-dimension spatial economy.

Fredriksson (2000) studies the political economy implications of different institutional structures on the choice of capacity of hazardous waste facilities in a federal system and finds that a decentralized system yields the first-best capacity level whereas a centralized system tends to implement sub-optimal levels. His paper does not take into account distance and no attention is paid to the choice of the (optimal) location of the facility, and to the interaction between (optimal) provision and location, which are instead the main objectives of our investigation. Kunreuther and Kleindorfer (1986) and Minehart and Neeman (2002) address the location problem of obnoxious facilities through auction-like procedures associated with compensation.

\footnotetext{
${ }^{4}$ Given this perspective, we leave aside the issue of optimal taxation policy which could affect the welfare consequences of the location of such a facility, by means of subsidies and taxes.
}

Facility location has also been extensively studied by operations research specialists. ${ }^{5}$ Our approach is in some ways similar to theirs, insofar as we explicitly take into account the distance between economic units (in our case, households) and the facility. ${ }^{6}$ However these approaches aim at finding efficient mechanisms and numerical algorithms to deal with the locational choice. Our approach innovates with respect to this literature in two ways: first we explore the trade-off between location and size, and we use a political economy perspective to tackle this trade-off.

The plan of this paper is as follows. Section 2 presents the model of a multi-household city facing the issue of the location and size of a public facility. In Section 3, we analyze the optimal solution of this problem made by a social planner. This solution will be used as a benchmark for the assessment of the political solution. Section 4 is devoted to the study of the political game when households lobby the policymaker in charge of the city. Section 5 concludes.

\section{The model}

We consider an economy formed of a territory and populated by $n$ households $(n>1)$. The territory is spatially defined as a convex compact $\mathcal{S} \subset \mathbb{R}^{2}$. We refer to this territory as the "city". A household $i$ is situated at one point $P_{i}$ in $\mathcal{S}$. $\left(x_{i}, y_{i}\right) \in \mathbb{R}^{2}$ are the coordinates of $P_{i}$. We denote by $\mathcal{P}$ the set of $n$ points $\left\{P_{1}, \ldots, P_{i}, \ldots, P_{n}\right\}$ and by $\tilde{\mathcal{P}}$ the convex hull of the $n$ points $\left\{P_{1}, . ., P_{i}, \ldots, P_{n}\right\}$.

A public facility has to be located in the territory $\mathcal{S}$. This facility provides a public good to agents. There exists a single policymaker who decides both on the (per-capita) size of the facility, measured in terms of the amount of the public good that provides, $g$, and its location, $L$, included in $\mathcal{S}$. The policymaker of the city, in charge of its public affairs, is called the "mayor". ${ }^{7}$ The location $L$ is fully characterized by its coordinates $\left(x_{l}, y_{l}\right)$ The Euclidean distance between $L$ and any point $P_{i}$ belonging to $\mathcal{P}$ is denoted by $d_{i}$.

The provision of the public good (equivalent to the size of the public facility) is financed by a lump-sum tax $\tau$. Assuming that there is no deficit, we have:

$g=\tau$

where $g$ denotes the per-capita provision of the public good. Each agent has the same endowment $e \in \mathbb{R}^{+}$and appreciates consuming the private and the public goods. In addition, the benefit she draws from the public good depends on the distance between her own location in the city and the location of the public facility.

Some facilities are such that any agent prefers them to be located as far as possible from her own location when they produce nuisances: think about landfills, hazardous waste facilities, jails, etc. Others, to the contrary, are most appreciated when they are close to individual: schools, museums, (underground) stations, etc., are examples of such facilities. We refer to the first ones as "Nimby" facilities and the second ones, by contrast, as "Imby" facilities.

Hence, for any agent $i$ belonging to $\mathcal{P}$, we write the utility function as follows:

$v_{i}\left(g, d_{i} ; e\right)=e-g+H(g)+F(g) K\left(d_{i}\right)$

with $H(0)=F(0)=0$ so that $v_{i}\left(0, d_{i} ; e\right)=e . H\left({ }^{\cdot}\right), F\left({ }^{\cdot}\right)$ and $K\left(\left(^{\cdot}\right)\right.$ are assumed to be continuously twice differentiable.

The first two terms correspond to net income equal to private consumption; the third term captures the benefits associated to the

\footnotetext{
${ }^{5}$ For an overview of the operations research literature on facility location, see for instance Drezner and Hamacher (2002) and Snyder (2006).

6 See, for example, Bhattacharya (2011) and Munoz-Perez and Saameno-Rodriguez (1999).

${ }^{7}$ Here what matters is that there is a closed unique political jurisdiction. The territory can also be seen as a "country" and its policymaker as the "government".
} 
consumption of the public good; and the last term corresponds to the impact of distance, that is the location effect. ${ }^{8}$ Private consumption enters linearly in the utility function as we want to focus on the sole location-size trade-off. With regard to the benefits of $g$ we will assume that $H(g)>0, H^{\prime}(g)>0$, with $H^{\prime}(0)>1, H^{\prime}(e)<1$, and $H^{\prime \prime}(g)<0$. $^{9}$

With regard to the location of the facility and its impact on a given household, the impact of distance is linked to the size (that is, the production of the public good) of the facility, as we shall discuss later. Here, let us focus on the impact of distance per se, which depends on whether the facility is Nimby or Imby. We assume that $K\left(d_{i}\right)$ is always positive and that:

$K^{\prime}\left(d_{i}\right)<0$.

We shall then formally define the two cases of interest, Nimby and Imby, as follows:

Definition 1. A Nimby (Imby) facility is such that $\frac{\partial v_{i}}{\partial d_{i}}>(<) 0$.

A simple way to distinguish between the two cases is related to the sign of $F(g): F(g)$ is negative in the case of a Nimby facility, and positive in the case of an Imby facility.

A Nimby facility generates spatial nuisances, that is, a reduction in the distance $d_{i}$ has a negative impact on individual utility. Moreover, a Nimby facility is characterized by the fact that agents prefer no facility at all when their distance to it is zero. We will incorporate this characteristic in our model by assuming that $\forall g \in(0, e]$ :

$v_{i}(g, 0 ; e)<e$.

To illustrate the case of a Nimby facility, consider a landfill generating unpleasant views and odors. The farther it is from one's location, the better it is for the agent. An Imby facility generates spatial benefits, that is, a reduction in the distance $d_{i}$ has positive impact on individual utility. As an example of an Imby facility, consider the case of the city hall. Distance is a proxy for the walking time to the city hall. The longer the walking time, the higher the opportunity cost it generates. Notice that the distance variable may capture both transportation costs and other non-pecuniary influences of distance on welfare. Here we do not disentangle the two effects.

In both cases, spatial effects are linked to the provision of the public good, that is the size of the facility supplying the public good. Defining the function $\Psi\left(g, d_{i}\right)$ as follows:

$\Psi\left(g, d_{i}\right)=F(g) K\left(d_{i}\right)$,

we shall refer to $g$ and $d_{i}$ as "complements" ("substitutes") for agent $i$ when the cross derivative $\Psi_{g d_{i}}\left(g, d_{i}\right)$ is positive (negative).

When $F^{\prime}(\cdot)<0, g$ and $d_{i}$ are complements. Then a decrease in the distance $d_{i}$ induces agent $i$ to ask for a decrease in the provision of the public good, as a diminished distance decreases the marginal benefit that agent $i$ draws from the public good. We refer to this case as the "congestion effect". ${ }^{10}$

When $F^{\prime}(\cdot)>0, g$ and $d_{i}$ are substitutes, and the opposite effect is at work: a decrease in distance augments the desirability of the public good for agent $i$. We refer to this effect as the "agglomeration effect".

Typically, we could think of airports as Nimby facilities with congestion effects. Ceteris paribus, the farther an airport is from residence, the bigger is its desired size (that is, the number of connections

\footnotetext{
8 This distance term may encompass various elements, such as tastes with respect to the properties of the public facility, congestion and transport costs when expressed in utility terms.

${ }^{9}$ Our assumptions on the derivatives of $H(\cdot)$ imply that, abstracting from the location effect, agents would have a most preferred level $g^{*} \in(0, e)$.

${ }^{10}$ In this case, as we have assumed that $F(0)=0$, it must be that: $\lim _{g \rightarrow 0+} F(g)<0$.
}

it offers to travelers): hence air travel and distance are complements. On the other hand, museums could be thought as Imby facilities with agglomeration effects between art and distance: the closer they are, the bigger is their desired size. This may help to explain why usually capitals enjoy the largest museums and large airports are located far away from the city center.

In sum, our specification of the individual utility function is fairly general. We introduce distance in the utility function, treating it as a public good per se, distinct from the amount of the public good provided to an agent. Not only can we formalize the direct effects of distance on individual utility, thus distinguishing Nimby goods from Imby goods, but also we can study the interaction between distance and provision, thus distinguishing a congestion effect from an agglomeration effect. As we shall see, this dual typology will prove quite effective in understanding the joint decision over size and location.

Finally, we introduce the following definition which will be useful in the sequel.

Definition 2. A n-household structure (n even) is O-symmetrical when, for any household located at point $P_{i}$, there exists another household located at point $P_{j}$ defined by $x_{j}=-x_{i}$ and $y_{j}=-y_{i}$ for $i=1, \ldots ., n$.

\section{The optimal solution}

Consider the problem solved by a benevolent social planner. Giving equal weight to all individuals, the social planner maximizes the average level of welfare. This solution will be used as a benchmark to assess the impact of lobbies on the political process leading to the location of the public facility. Taking into account Eq. (2), the planner solves the following optimization problem:

$$
\begin{aligned}
& \max _{g, x_{l}, y_{l}} W\left(g, x_{l}, y_{l} ; e, x_{i}, y_{i}\right)=[e-g+H(g)]+F(g)\left[\frac{1}{n} \sum_{i=1}^{n} K\left(d_{i}\right)\right] \\
& \text { s.t. } \quad d_{i}=\left[\left(x_{i}-x_{l}\right)^{2}+\left(y_{i}-y_{l}\right)^{2}\right]^{\frac{1}{2}} \\
& \left(x_{l}, y_{l}\right) \in \mathcal{S} \\
& d_{i} \geq 0 \\
& g \in[0, e] .
\end{aligned}
$$

It is easy to prove the existence of an optimal solution denoted by $\left(g^{*}, x_{l}^{*}, y_{l}^{*}\right)$. It comes from the continuity of welfare function and compactness of the opportunity set, defined in terms of available spatial and physical resources. ${ }^{11}$ Notice that, given separability of $g$ and $d$ in the utility function, we can solve the optimal location problem separately from the optimal provision problem. Starting with the former, we can state the following:

\section{Proposition 1. The optimal location}

(i)The optimal location is such that $d_{i}^{*} \geq 0$ with $d_{i}^{*}=0$ for at most one $i$. (ii) If the facility is Imby, the optimal location $L^{*}=\left(x_{l}^{*}, y_{l}^{*}\right)$ is unique if $K^{\prime \prime}\left(d_{i}\right)<0, \forall d \in \mathbb{R}^{+}$. Moreover, $L^{*}$ belongs to $\tilde{\mathcal{P}}$. (iii) If the facility is Nimby, the optimal location $L^{*}=\left(x_{l}^{*}, y_{l}^{*}\right)$ is unique if $\frac{K^{\prime \prime}\left(d_{i}\right) d_{i}}{K^{\prime}\left(d_{i}\right)}<-1$, $\forall d \in \mathbb{R}^{+}$. Moreover, if $L^{*}$ does not belong to the interior of $\tilde{\mathcal{P}}$, then it is on the boundary of $\mathcal{S}$.

\footnotetext{
11 Formally, we define $z \equiv\left(g, x_{l}, y_{l}\right) \in \mathcal{Z}$ where $\mathcal{Z}$ is a non-empty subset of the Euclidean space $E^{3}$. Given that $\mathcal{S}$ is compact and $g$ is finite, $W$ is continuous on $\mathcal{Z}$ and, according to the Weierstrass theorem, there exists a global maximum which is either interior or on the boundary of $\mathcal{Z}$.
} 


\section{Proof. See appendix A.}

A key feature of the optimal solution is that, for any kind of facility, be it Nimby or Imby, uniqueness of the equilibrium is not guaranteed unless additional conditions are imposed on the distance term in the utility function. It is easy to construct distributions of neighborhoods, for example symmetrical ones, such that multiple solutions arise. For instance, suppose that there are two neighborhoods located at $(1,0)$ and $(-1,0)$; then the optimal location of a Nimby facility, because of the symmetrical characteristics of the problem, is either $(0,1)$ or $(0,-1)$.

A linear specification of $K(\cdot)$ would also generate multiple solutions, given a distribution of neighborhoods on the territory. ${ }^{12}$

In the Imby case, a standard concavity assumption on $K\left({ }^{\cdot}\right)$ is sufficient to ensure uniqueness. Suppose there are two neighborhoods. Since the benefit of distance is decreasing at a decreasing rate, locating the facility just in the middle of the segment linking the two neighborhoods maximizes the social benefit of the facility. This reasoning easily generalizes to more than two agents.

In the Nimby case however, the concavity assumption is no longer sufficient for uniqueness. ${ }^{13}$ In addition to this assumption, the elasticity of individual utility with respect to distance $\frac{K^{\prime \prime}\left(d_{i}\right) d_{i}}{K^{\prime}\left(d_{i}\right)}$ has to be sufficiently small. To get the intuition, let us suppose to start from an optimal location and ask: is it possible to find another location such that some individuals benefit and some other individuals lose, yet the social welfare is kept constant? The elasticity condition rules out this possibility, as the loss suffered by some neighborhood (to which the new location is closer) is sufficiently large and the benefits enjoyed by some other neighborhood (from which the new location is farther away) is sufficiently small. The elasticity condition ensures that the new location cannot generate the same level of social welfare of the initial optimal solution.

This difference between Nimby and Imby facilities may explain why there are more outcries and protests in the case of the location of the former ones than in the case of the latter ones. Most people will agree that locating the town hall in the center of the city, or the National Museum of Fine Arts (as examples of Imby goods) in the capital is socially optimal. Consequently, not many object to this choice. However, this is not true for Nimby facilities. The sufficient condition for a unique socially optimal being more difficult to meet, there is no "obvious" choice for a waste facility. Hence the concerned neighborhoods fight and attempt to influence the location choice of the policymaker as no one is obviously better.

When the public facility is Imby, and the solution is unique, the facility is located within the convex set $\tilde{\mathcal{P}}$. Suppose it is not the case. Then, moving orthogonally toward the convex hull will make everybody better off, at least until the boundary of the hull is reached. All individuals agree on the fact that the public facility must be as close as possible from their own location. This explains why the optimal location lies within the hull formed by the households.

When the public facility is Nimby, it is easy to understand that, if the optimal location is located outside the convex hull $\tilde{\mathcal{P}}$, it must lie on the boundary of $\mathcal{S}$. Suppose it is not the case. Then, moving orthogonally the public facility further away toward the boundary will make all agents better off.

\footnotetext{
12 When $K(\cdot)$ is linear, the problem reduces to maximization (minimization) of the sum of distances (i.e. average distance) in the Nimby (Imby) case. Consider the following example for the Imby case. Two households $P_{1}$ and $P_{2}$ are located at $(1,0)$ and $(-1,0)$ respectively. It is immediate to see that any location on the line which connects the two households would minimize the sum of distances, and the average distance is equal to 1 . In the Nimby case, assuming that the two households are located as in the previous example, it is also easy to see that optimal facility location is either $(0,1)$ or $(0,-1)$ and the average distance is $\sqrt{2}$.

${ }^{13}$ Notice that, in the Nimby case, concavity of the distance term implies $K^{\prime \prime}(\cdot)>0$ as $F(g)<0$.
}

Let us turn now to the properties of the optimal size of the facility, $g^{*}$. Here, we can state the following:

\section{Proposition 2. The optimal size}

(i) In the case of a Nimby good, a corner solution may arise such that $g^{*}=0$. (ii) Let $\mathcal{K} \equiv \frac{1}{n} \sum_{i=1}^{n} K\left(d_{i}\right)$ denote the "average distance impact" on utility. Consider an interior solution $g^{*} \in(0, e)$. Then, $g^{*}$ is an increasing function of $\mathcal{K}$ if and only if size and distance are substitutes, that is if and only if $F^{\prime}(g)>0$.

\section{Proof. See Appendix B.}

When the good is Imby, any $g>0$ is preferred by agents to $g=0$. When the good is Nimby, zero provision can be optimal as the positive consumption effect is traded against the negative location effect. Notice that the fact that $g^{*}$ can be equal to zero is a consequence of our definition of Nimby goods, as expressed in Eq. (3). ${ }^{14}$ In other words, Eq. (3) is a necessary condition for this corner solution to arise. In fact, if even the agent located at zero distance from the facility prefers a positive level of the public good, a fortiori the social planner, who maximizes utility of the "average" individual, would choose a positive level of $g$. Clearly, the larger is the average distance impact $\mathcal{K}$, the more likely such a corner solution occurs. ${ }^{15}$

The second part of this proposition emphasizes the interdependence between size and location, stressing the role of the complementarity or substitutability property of distance and size in the utility function. This happens because the spatial externalities depend on the amount of public good supplied to the city's population.

When the average distance impact increases, and $g$ and $d$ are substitutes, the marginal benefit of an increase in $g$ increases. If we start from an interior solution (which implies concavity of the utility function), the optimal size also increases. The opposite is true when $g$ and $d$ are complements: the marginal benefit of $g$ decreases so that the optimal size decreases.

In brief, the provision of a public good in an economy where agents are dispersed on a non-degenerate territory depends on the characteristics attached to the location of the facility producing this public good in the territory. This link crucially depends on two features. The first one is the direct effect of distance, what we could call the "(n)imbyness of the facility". The second one is the cross-effect of distance upon the benefits attached to the amount of available public good, that is, whether there is a congestion effect or an agglomeration effect.

\section{Lobbying on location}

We now introduce the possibility that households lobby the mayor for the location and size of the public facility. We will formalize the lobbying process as a common agency game à la Bernheim and Whinston (1986), where lobbies offer binding contributions to the mayor, conditional on the chosen policy.

Let us denote with $\mathcal{L} \subseteq \mathcal{P}$ the subset of $\mathcal{P}$ whose elements (households) are lobbying and with $l$ the cardinal of $\mathcal{L}: l \leq n$. We refer to $\mathcal{L}$ as the "active set" and to $\overline{\mathcal{L}}$, the complement of $\mathcal{L}$ in $\mathcal{P}$, as the "passive set".

The lobbying game on the location and provision of the public good is similar to Grossman and Helpman's (1994) analysis of trade policy. It is a two-stage game.

1. In the first stage, lobbies commit to a menu of contributions depending on the policy chosen by the mayor. Given Eq. (2), individual contribution schedule by lobby $i$ is a function of $g$ and $d_{i}$ and is denoted by $C_{i}\left(g, d_{i}\right)$. It is assumed to be globally truthful, that is $C_{i}(g$,

\footnotetext{
${ }^{14}$ We would like to thank an anonymous referee for pointing out this to us.

15 Notice that in principle it would be possible that $\tilde{d}_{i}^{*}=0$ for one $i$ and $g^{*}>0$. This can happen as long as the disutility suffered by agent $i$ is more than compensated by the utility enjoyed by all other agents.
} 
$\left.d_{i}\right)=\max \left[0, v\left(g, d_{i} ; e\right)-b_{i}\right]$ where $b_{i}$ is a scalar optimally set by lobby $i$. Lobbies play non-cooperatively with one another: when choosing

$b_{i}$, each lobby takes other lobbies' contributions as given.

2. In the second stage, the mayor decides on the location and size of the public facility, taking into account the related contributions that she will receive from the various lobbies. The maximization problem of the mayor in the presence of lobbies is written as:

$\max _{g, x_{l}, y_{l}} \Xi\left(g, x_{l}, y_{l}\right) \equiv \lambda W\left(g, x_{l}, y_{l}\right)+(1-\lambda) \sum_{i \in \mathcal{L}} C_{i}\left(g, d_{i}\right)$

where $W\left(g, x_{l}, y_{l}\right)$ is the social welfare function given in Eq. (6), and $\lambda \in[0,1]$ is the weight given to social welfare and is an index of "benevolence". When $\lambda$ is equal to 1 , the mayor acts as the social planner and implements the optimal solution characterized in the previous section; when it is equal to zero, the mayor is fully opportunistic.

A solution to the lobbying game is a vector $\left(\hat{g}, \hat{x}_{l}, \hat{y}_{l}, \hat{c}_{1} \ldots, \hat{c}_{i}, . ., \hat{c}_{n}\right)$ where $\hat{c}_{i}$ is the contribution received by the mayor from neighborhood $i$, associated with $\left(\hat{g}, \hat{x}_{l}, \hat{y}_{l}\right) .{ }^{16}$ We denote by $\hat{L}=$ $\left(\hat{g}, \hat{x}_{l}, \hat{y}_{l}, \hat{c}_{1} \ldots, \hat{c}_{i}, . ., \hat{c}_{n}\right)$ the location associated with this solution.

Then, we can state the following result on this lobbying game:

Proposition 3. The lobbying game equilibrium

The lobbying game equilibrium replicates the properties of the social planner solution in terms of existence and uniqueness. Moreover, the amount of contribution $\hat{c}_{i}$ is increasing (decreasing) in $\hat{d}_{i}$ if the facility is Nimby (Imby), for all $i$.

\section{Proof. See Appendix C.}

The existence of a solution is immediate, given the standard features of this economy and the assumption of truthful contributions. Conditions for uniqueness can be understood using the same reasoning made for Proposition 1 in the case of the social planner solution.

Insofar as the location of the facility is concerned, we can apply the same arguments that we used for Proposition 1. For example, in the Nimby case, if the public facility were located outside the convex hull $\tilde{\mathcal{P}}$ but not on the boundary, all agents (active and passive) would agree on moving the facility orthogonally to the boundary and the mayor would implement this move. In this case, there would be no conflict of interest whatsoever between lobbies and non-lobbies.

According to Eq. (2), household $i$ 's utility depends only on her distance to the public facility and on the amount of the public good. In the Nimby case, the more distant is the public facility to agent $i$, the higher is her level of utility. By truthfulness assumption, contributions must reflect exactly the relative valuation of two alternatives, so that agent $i$ must bid more the more distant is the facility. The opposite holds for the Imby case.

Assuming for simplicity that the conditions for a unique solution are satisfied both for the social planner's problem and for the lobbying game, ${ }^{17}$ we can prove the following:

\section{Proposition 4. The impact of lobbies}

(i) If $\mathcal{L}=\mathcal{P}$, the set of equilibrium locations $L^{*}$ is identical to the set of optimal locations $L^{*}$ and the size $\hat{g}$ associated to a particular location is

\footnotetext{
${ }^{16}$ If household $i$ belongs to the passive set, its contribution $H(\cdot)$ is automatically equal to 0 .

17 In the case of multiple optimal solutions, we would have to use a selection criterion in order to make comparisons. An obvious criterion would to focus on the optimal solution(s) for which the welfare of the lobby set is maximized. Our analysis would then proceed.
}

identical to the size $g^{*}$ chosen by the social planner. (ii) If $\mathcal{L} \subset \mathcal{P}$ it may be the case that $\hat{L}=L^{*}$. (iii) When $\hat{L} \neq L^{*}$, and the good is Nimby (Imby), $\hat{g}>$ $g^{*}$ if and only if $g$ and $d$ are complements (substitutes).

\section{Proof. See Appendix D.}

Result (i) is a well-known property of this type of lobbying games: when all households lobby, the solution is identical to the optimal one. In our case, it means that both the location and size of the public facility are equal to the ones chosen by the social planner. When all households lobby, their actions nullify each other and the countervailing power of each lobby against all lobbies leaves the mayor in a position to choose the socially optimal solution. Of course, in this case there is no net gain in lobbying. For instance, by Propositions 1 and 2 and Definition 2, if $\mathcal{L}=\mathcal{P}$, and $\mathcal{P}$ is $O$-symmetrical, the center of the city may well be the solution of the lobbying game, whether the public facility is Nimby or Imby. In that case, given the symmetric location of all households with respect to $O$, they would all contribute the same positive amount.

Result (ii) contrasts with the result obtained by Grossman and Helpman (1994). In their model, the solution when only a subset of agents lobby always differs from the solution when all agents lobby. Here, the optimal solution may be obtained even though not all neighborhoods lobby. A simple example will provide the intuition for this result. Consider an $\mathrm{O}$-symmetrical structure such that the social planner's optimal location is $O$. Suppose that only households $P_{i}$, with $i$ even, are active lobbies. Then each lobby faces a symmetrical lobby with respect to $O$. The lobbying game replicates the $O$-symmetry property of the social planner case, and the mayor locates the public facility in 0 . It happens that each lobby will neutralize the action of its counterpart.

To illustrate this result, we rely here for simplicity on a symmetry assumption. However, it is possible to construct examples with non symmetrical lobbies which would generate the result that the optimal location is the solution of the lobbying game. This result does not depend on some stringent geometrical assumptions over the distribution of agents on the territory.

This result may provide an explanation for the empirical finding by Feinerman et al. (2004). In their empirical study of the siting of a waste disposal facility in Israel, they found that the actual choice made by the Israeli authorities almost coincides with the "optimal" solution that would have been chosen by the social planner. This is likely to be the consequence of the countervailing influences of the diverse lobbies involved in the decision making process.

With regard to result (iii), it illustrates the suboptimality of the lobbying solution when only some households are able to influence the government.

To understand the latter result, consider the simple case of a Nimby facility with a single lobbying household, $P_{1}$, with $F^{\prime}(g)<0$. In this case, we know that the public good and distance are complements. The lobbying household, having no countervailing household, is able to induce the mayor to locate the public facility farther from itself, thereby decreasing the negative spatial externalities suffered by $P_{1}$. Then, as the distance is increased, $P_{1}$ is willing to benefit from a larger amount of the public good, because her marginal benefit from public good consumption, given by $H^{\prime}(g)-1+F^{\prime}(g) K\left(d_{1}\right)$ increases with $d_{1}$, as $K\left(d_{1}\right)$ decreases. This explains why the mayor is induced by the pressure of $P_{1}$ to choose $\hat{g}$ larger than the socially optimal $g^{*}$.

On the contrary, if the public good and distance are substitutes, the marginal benefit of public good consumption decreases when distance is increased. Thus the mayor is induced to choose $\hat{g}$ smaller than the socially optimal $g^{*}$.

In the case of an Imby facility, a similar reasoning applies. Again suppose that $P_{1}$ is the unique lobbying household, with $F^{\prime}(g)>0$, so that $g$ and $d$ are substitutes. She wants the public facility to be located 
closer to her. But then, as the distance is decreased, the marginal benefit that she draws from the public good increases. Therefore she is willing to redirect some resources to the public good, and the mayor is induced by her contribution schedule to increase the amount $\hat{g}$ compared to the socially optimal $g^{*}$. The opposite would happen if $g$ and $d$ were complements. ${ }^{18}$

In short, as it was true in the case of the decisions of a social planner, the political decisions on the location of a facility and its size are intimately related. The two structural features governing this link are the nature of the facility (Nimby or Imby) and the existence of a congestion effect or an agglomeration effect. As a consequence, under-provision or over-provision of the public good can occur in both types of facilities, that is, when the political location differs from the optimal one.

Turning to the welfare properties of the lobbying solution, we can show that, on this dimension too, the impact of lobbies may be paradoxical. Again, for simplicity, we restrict the analysis to the case where there is a unique solution to the social planner problem and to the lobbying game. Then we can prove:

\section{Proposition 5. Lobbies and welfare}

When $\mathcal{L} \subset \mathcal{P}$, and $\hat{L} \neq L^{*}$ :

(i) $\sum_{\left(g_{i \in \mathcal{L}}\right.} v_{i}\left(\hat{g}, \hat{d}_{i}\right)>\sum_{i \in \mathcal{L}} v_{i}\left(g^{*}, d_{i}^{*}\right)$ and $\sum_{i \in \overline{\mathcal{L}}} v_{i}\left(\hat{g}, \hat{d}_{i}\right)<\sum_{i \in \overline{\mathcal{L}}} v_{i}$ $\left(g^{*}, d_{i}^{*}\right)$

(ii) there may exist some households $P_{i}$ belonging to $\mathcal{L}$ such that $v_{i}\left(\hat{g}, \hat{d}_{i}\right)<v_{i}\left(g^{*}, d_{i}^{*}\right)$ and households $P_{j}$ belonging to $\overline{\mathcal{L}}$ such that $v_{j}\left(\hat{g}, \hat{d}_{j}\right)>v_{j}\left(g^{*}, d_{j}^{*}\right)$.

\section{Proof. See Appendix E.}

This proposition characterizes the welfare properties of the solution when $\mathcal{L} \subset \mathcal{P}$, and the mayor's decision differs from the optimal solution. Property (i) states that, taken collectively, the active households benefit from this decision (not taking into account their contributions), at the expense of the passive households: altogether the active households are better off with the decision reached when they lobby than with the solution chosen by the social planner. The reverse is true for the passive households.

However it is not true that each single lobby benefits from the mayor's decision, nor that each passive household is harmed by it (property (ii)). Two simple examples will help to explain these counter-intuitive results. Assume $F(g)=g$ and consider a $O$-symmetrical structure, with $n$ households, $n$ being large. The public facility is Nimby. Suppose that all households $P_{i}, i \leq n / 2$, are active lobbies and that among the rest of households, there is only one active lobby, $P_{j}, j>n / 2$. The $n / 2$ first lobbies have a common interest to locate the public facility on the down-half of the trigonometric circle (with negative $y$ ). This harms household $P_{j}$ as this shortens the distance $d_{j}$.

Suppose now that there are only two active lobbying households, $P_{i}$ and $P_{i+2}$. Their interests coincide and they act so as to increase the distance $d_{i}$ and $d_{i+2}$. But, by so doing, they contribute to increase the distance $d_{i+1}$ thereby benefitting the in-between passive household $P_{i+1}$.

Proposition 5 can be better understood by contrasting it with the results obtained by Grossman and Helpman (1994). In their model, the pecuniary externalities generated by any contributing lobby on any other agent (lobby or not) is always negative: since all goods are consumed by all agents, rising tariff on one good through the lobbying activity of its producer always harms all other agents. In contrast, in our framework, considering as an example the case of a

\footnotetext{
18 Notice that these examples can be generalized to the case of a plurality of lobbies
}

Nimby facility, increasing the distance between one lobbying household and the location of the public facility may benefit other households, provided they are close enough to the lobby's location. In other words, differently from Grossman and Helpman (1994), here there may be a congruence in interests among different neighbors. Moreover, as the example developed in the Appendix makes clear, this result does not depend on a symmetry assumption. This may happen for a distribution of numerous households on the city's territory quite skewed.

The last paragraph sheds light on the free riding strategies being played in both settings. In both models, there is an incentive to defect from the solution without contributions: if no agent is actively lobbying, any agent has an incentive to be an active lobby. However, in Grossman and Helpman (1994) when other lobbies increase their contributions, any lobby is induced to increase hers because of the negative externalities, whereas in our case, due to the possible convergence of some agents' interests, a lobbying household may have an incentive to free ride on her close neighbors and decrease her contributions when theirs are increased.

\section{Conclusion}

This paper tackles the joint issue of the location and size of a public facility in a territory from a theoretical perspective. We offer a specification of the individual utility function which allow us to distinguish two properties attached to the location of a public facility. One is the "nimbyness" of the facility: whether an increase in the distance from the facility affects the welfare of an agent positively or negatively. The second is linked to the interacting effects of location and size, that is the amount of the public good provided to an agent: The public good and distance are "complements"("substitutes") when a decrease in distance decreases (increases) the marginal benefits that individuals enjoy from an increase in the provision of the public good. In the first case, we refer to a "congestion effect", and, in the latter case, to an "agglomeration effect".

Considering a two-dimensional territory which we call a city, we have characterized the optimal solution chosen by a social planner, which we take as a benchmark. The uniqueness of the optimal decision is not guaranteed neither in the Nimby case nor in the Imby one. We infer from this result that the debates about the location of a facility are likely to be acrimonious, as the multiplicity problem has to be overcome. The optimal provision of the public good is related to the optimal location of the facility. Both the "nimbyness" of the facility and the nature of the public good-distance nexus matter for the optimal choice of the size of the public facility.

Then, adopting a political economy perspective on the subject, we have analyzed a lobbying equilibrium à la Grossman and Helpman (1994), showing that this equilibrium can replicate the optimal solution not only when all households lobby the "mayor", but also when only a subset of households is actively involved in lobbying activities. When the mayor's decision differs from the optimal solution, it may lead to either over- or under-capacity of the public facility. When some households are organized in lobbies, there is inefficient provision of the public good. The direction of the inefficiency (under-provision or over-provision) depends on the nature of the good and whether the public good and distance are substitutes or complements. Additionally, there is again the possibility of multiple solutions.

Turning to the normative analysis of the political game, whereas as a whole lobbies gain from the equilibrium game, and non-lobbies lose, it may happen that some lobbies lose despite their own political involvement and some non-lobbies gain despite their inactivity. This raises the issue of who should lobby and who should not.

Our analysis rests on some simplifying assumptions, which might be relaxed to shed light on new issues related to the location decision of a public facility. 
Here the players of our political game are exogenously given. In particular we do not consider the lobbying decision as such. ${ }^{19}$ Similarly we do not address the issue of the selection of the policymaker, the "mayor". A city is the locus of an active political life and competition which cannot be reduced to the actions of lobbies towards an a-temporal mayor. It would be worth to relate (local) democracy to the location issue of a public facility and investigate how this issue impinges on the electoral competition and selection process. ${ }^{20}$

Regarding the specification of the model, various modifications could be introduced. A first source of complexity would be to assume that households have different tastes such that some would consider the public facility as Nimby and others as Imby. Another one would be to consider a multi-jurisdictional territory with transboundary effects of public facilities. ${ }^{21}$

We assume that there is no individual decision on private consumption and/or productive activities. Of course, a household may modify his own private decisions, depending on the location and size of the public facility. Studying this problem requires a slightly more complex model than the one developed here.

Lastly, a central dimension of the location of a public facility is the issue of its impact on the location of private production factors. The interplay between the public decision of locating a public facility in a given territory and the private decisions of locating private production factors deserves to be investigated in a political economy perspective. $^{22}$

We leave these intriguing issues to future research.

\section{Appendix A. Proof of Proposition 1}

(i) Obviously, the public facility can be located at the exact location of at most one household. (ii) As discussed in the paper, given the objective function of the planner in Eq. (6), we can separate the optimal location problem from the optimal size problem. In Proposition 1, we deal with the optimal location problem which is independent of the level of $g$. Let:

$\Psi\left(g, d_{i}\right)=F(g) K\left(d_{i}\right)$.

The Hessian associated with $\Psi$ is:

$\operatorname{Hess}(\Psi)=F(g)\left(\begin{array}{ll}\frac{K^{\prime \prime}\left(d_{i}\right) \cdot\left(x_{l}-x_{i}\right)^{2}}{d_{i}^{2}}+\frac{K^{\prime}\left(d_{i}\right) \cdot\left(y_{l}-y_{i}\right)^{2}}{d_{i}^{3}} & \left(\frac{K^{\prime \prime}\left(d_{i}\right)}{d_{i}^{2}}-\frac{K^{\prime}\left(d_{i}\right)}{d_{i}^{3}}\right)\left(x_{l}-x_{i}\right)\left(y_{l-y_{i}}\right) \\ \left(\frac{K^{\prime \prime}\left(d_{i}\right)}{d_{i}^{2}}-\frac{K^{\prime}\left(d_{i}\right)}{d_{i}^{3}}\right)\left(x_{l}-x_{i}\right)\left(y_{l}-y_{i}\right) & \frac{K^{\prime \prime}\left(d_{i}\right) \cdot\left(y_{l}-y_{i}\right)^{2}}{d_{i}^{2}}+\frac{K^{\prime}\left(d_{i}\right) \cdot\left(x_{1}-x_{i}\right)^{2}}{d_{i}^{3}}\end{array}\right)$.

Its trace is equal to:

$\operatorname{tr}=F(g)\left[K^{\prime \prime}\left(d_{i}\right)+\frac{K^{\prime}\left(d_{i}\right)}{d_{i}}\right]$

and the determinant is given by:

$\operatorname{det}=F(g)\left[\frac{K^{\prime \prime}\left(d_{i}\right) K^{\prime}\left(d_{i}\right)}{d_{i}}\right]$.

\footnotetext{
19 Some papers study the endogenous decision to lobby in different contexts (see for example Felli and Merlo (2006) and Mitra (1999)). Endogenizing lobbying activities would also raise important issues related to coalition formation. The coalitionproofness of the truthful contributions equilibrium, proven by Bernheim and Whinston (1986), would then play a crucial role.

${ }^{20}$ Besley and Coate (2001) have proved in a citizen-candidate model of democracy that the electoral process limits drastically the influence capacity of lobbies.

${ }^{21}$ For an investigation on the siting of obnoxious facilities and polluting firms in a multi jurisdictional setting, see Besfamille and Lozachmeur (2010), Ingberman (1995) and Wellisch (1995).

22 See Thisse and Wildasin (1992) and Ottaviano and Thisse (2002) for explorations of this dimension of competition between communities.
}

Notice that $\operatorname{tr}<0$ and $\operatorname{det}>0$ are sufficient conditions for strict concavity of $\Psi\left(g, d_{i}\right)$.

(i) The Imby case.

In the Imby case, as $F(g)>0$, if $K^{\prime \prime}\left(d_{i}\right)$ is negative, the trace is negative and the determinant is positive (remember that $K^{\prime}\left(d_{i}\right)<0$ ). Hence, if $K^{\prime \prime}\left(d_{i}\right)$ is negative, $\Psi\left(g, d_{i}\right)$ is strictly concave in $\left(x_{l}, y_{l}\right)$. Since the sum of (strictly) concave functions is (strictly) concave, the sufficient condition for the existence of a unique optimal location $L^{*}=\left(x_{l}^{*}, y_{l}^{*}\right)$ is satisfied.

To prove the second part of (i), suppose that $L^{*}$ does not belong to $L^{*}$. Let $\tilde{\mathcal{P}}$ denote the orthogonal projection of $L^{*}$ on $\tilde{\mathcal{P}}$. Then, the point $\tilde{L}$ is nearer than $L^{*}$ to any $P_{i}$, that is:

$\left\|L^{*}-P_{i}\right\|>\left\|\tilde{L}-P_{i}\right\|, \forall i$

so that:

$W\left(g^{*}\left(L^{*}\right), \tilde{L}\right)>W\left(g^{*}\left(L^{*}\right), L^{*}\right)$

(ii) The Nimby case.

Recall that, in this case, $F(g)<0$. Thus, $K^{\prime \prime}\left(d_{i}\right)>0$ is sufficient and necessary condition for the determinant to be positive. However, this does not guarantee that the trace is negative, as $K^{\prime}\left(d_{i}\right)<0$. For the trace to be negative, it has to be the case that $K^{\prime \prime}\left(d_{i}\right)+\frac{K^{\prime}\left(d_{i}\right)}{d_{i}}>0$ which can be rewritten as $\frac{K^{\prime \prime}\left(d_{i}\right) d_{i}}{K^{\prime}\left(d_{i}\right)}<-1$ We can thus conclude that the latter inequality (which implies $K^{\prime \prime}\left(d_{i}\right)>0$ ) is a sufficient condition for strict concavity of $\Psi\left(g, d_{i}\right)$ in the Nimby case, which implies that there exists a unique optimal location $L^{*}=\left(x_{l}^{*}, y_{l}^{*}\right)$.

To prove the second part of (ii), suppose that $L^{*}$ does not belong to the interior of $\tilde{\mathcal{P}}$ nor to the boundary of $\mathcal{S}$. Let $\tilde{L}$ denote the orthogonal projection of $L^{*}$ on $\tilde{\mathcal{P}}$. Consider the point $C^{*}$ located on the boundary of $\mathcal{S}$, and such that its orthogonal projection on $\tilde{\mathcal{P}}$ is the same point $\tilde{L}$. Then, the point $C^{*}$ is more distant than $L^{*}$ for any $P_{i}$, that is:

$\left\|C^{*}-P_{i}\right\|>\left\|L^{*}-P_{i}\right\|, \forall i$

so that:

$W\left(g^{*}\left(L^{*}\right), C^{*}\right)>W\left(g^{*}\left(L^{*}\right), L^{*}\right)$.

It is easy to construct an example where the optimal location belongs to the interior of $\tilde{\mathcal{P}}$. Consider four households forming a square within a circle of ray 1 . Suppose that

$v_{i}\left(g, d_{i} ; e\right)=e-g+H(g)-g d_{i}^{-1}$.

That is, $F(g)=-g$ (the facility is Nimby) and the function $K(\cdot)=d_{i}^{-1}$. Then the optimal solution is the center of the circle, where diagonals intersect. $^{23}$

\section{Appendix B. Proof of Proposition 2}

(i) For a corner solution $g^{*}=0$ to arise it must be the case that $W^{\prime}(0) \leq 0$ which can happen only if $F^{\prime}(0)<0$ given our assumption that $H^{\prime}(0)>1$. Let us we provide two numerical examples to show that both corner and interior solutions can occur depending on functional forms and parameter values. Consider first the following example: $H(g)=-\frac{1}{3} g^{2}+\frac{11}{10} g$, $F(g)=-2 g^{\frac{1}{2}}, n=5, e=1$ and $\sum K\left(d_{i}^{*}\right)=1$. It is immediate to

\footnotetext{
${ }^{23}$ We are very indebted to Stephane Rossignol for the proof of this proposition.
} 
check that the optimal solution in this case is $g^{*}=0$. Consider next that $H(g)=-\frac{1}{3} g^{2}+7 g, F(g)=-2 g^{2}, n=5, e=10$ and $\sum K\left(d_{i}^{*}\right)=1$. In this case, it is easy to check that the optimal solution is $g^{*} \simeq 4.09$. Notice that in this case our definition of Nimby is satisfied as long as $K(0)>2.13$.

(ii) For a given optimal location, the optimal provision of public good, if interior, solves $H^{\prime}\left(g^{*}\right)-1+F^{\prime}\left(g^{*}\right)\left[\frac{1}{n} \sum_{i=1}^{n} K\left(d_{i}^{*}\right)\right]=0$. In order to ensure that $g^{*}$ is a global maximum, the second-order condition must be satisfied, that is:

$$
H^{\prime \prime}(g)+F^{\prime \prime}(g)\left[\frac{1}{n} \sum_{i=1}^{n} K\left(d_{i}^{*}\right)\right]<0 .
$$

The last inequality will be satisfied whenever:

$$
F^{\prime \prime}(g)<-\frac{H^{\prime \prime}(g)}{\left[\frac{1}{n} \sum_{i=1}^{n} K\left(d_{i}^{*}\right)\right]} \text {. }
$$

A sufficient condition for the latter inequality to hold is $F^{\prime \prime}(g)<0$.

Denoting by $\mathcal{K}$ the average distance impact $\left[\frac{1}{n} \sum_{i=1}^{n} K\left(d_{i}\right)\right]$ and differentiating the first order condition with respect to $g$, it yields:

$\frac{d g}{d \mathcal{K}}=-\frac{F^{\prime}(g)}{H^{\prime \prime}(g)+F^{\prime \prime}(g) \mathcal{K}}$

By the second order condition of the maximization problem, the denominator is negative. Thus, $\frac{d g}{d \mathcal{K}} \gtrless 0 \Leftrightarrow F^{\prime}(g) \gtrless 0$.

\section{Appendix C. Proof of Proposition 3}

Given our assumptions, the maximization problem of the mayor given in (11) reduces to:

$\max _{g, x_{l}, y_{l}} \lambda \sum_{i \in \mathcal{L}} v_{i}\left(g, d_{i}\right)+\sum_{i \in \mathcal{L}} v_{i}\left(g, d_{i}\right)$

that is, the weight of non lobbies in the social welfare function is equal to $\lambda$ which is smaller than the weight associated to lobbies, which is equal to one. Then the proof of the first part of the proposition (existence and characterization of the equilibrium location) proceeds as in the proof of Proposition 1.

To prove the second part of the proposition, let $(g, L)$ and $\left(g^{\prime}, L^{\prime}\right)$ denote two possible vectors of location of the public facility and allocation of the public good and assume that $d_{i}>d^{\prime}{ }_{i}$. Then, for agent $i, v_{i}(g$, $\left.d_{i}\right)>(<) v_{i}\left(g^{\prime}, d_{i}^{\prime}\right)$ in the Nimby (Imby) case. By the definition of truthful contributions, agent $i$ must offer more (less) for $L$ than for $L^{\prime}$ if the good is Nimby (Imby).

\section{Appendix D. Proof of Proposition 4}

(i) If $\mathcal{L}=\mathcal{P}$, the maximization problem of the mayor in Eq. (17) becomes:

$$
\max _{g, x_{l}, y_{l}} \sum_{i \in \mathcal{P}} v_{i}\left(g, d_{i}\right)
$$

which is exactly the same problem that is solved by the social planner.

(ii) We know from (i) that when $\lambda=1, \hat{g}=g^{*}$. Moreover, we can calculate $\frac{d \hat{g}}{d \lambda}$ :

$$
\begin{gathered}
\frac{d \hat{g}}{d \lambda}=-\frac{F^{\prime}(g)\left[\sum_{i \in \mathcal{L}} \frac{\partial K\left(d_{i}\right)}{\partial d_{i}}\left(\frac{\partial d_{i}}{\partial \hat{x}} \frac{\partial \hat{x}}{\partial \lambda}+\frac{\partial d_{i}}{\partial \hat{y}} \frac{\partial \hat{y}}{\partial \lambda}\right)+\hat{D}_{\overline{\mathcal{L}}}^{-}+\lambda \sum_{i \in \overline{\mathcal{L}}} \frac{\partial K\left(d_{i}\right)}{\partial d_{i}}\left(\frac{\partial d_{i}}{\partial \hat{x}} \frac{\partial \hat{x}}{\partial \lambda}+\frac{\partial d_{i}}{\partial \hat{y}} \frac{\partial \hat{y}}{\partial \lambda}\right)\right]}{H^{\prime \prime}(\hat{g})+F^{\prime \prime}(g)\left[\lambda \hat{D}_{\overline{\mathcal{L}}}^{-}+\hat{D}_{\mathcal{L}}\right]} \\
+\frac{H^{\prime}(\hat{\mathrm{g}})-1}{H^{\prime \prime}(\hat{\mathrm{g}})+F^{\prime \prime}(g)\left[\lambda \hat{D}_{\overline{\mathcal{L}}}^{-}+\hat{D}_{\mathcal{L}}\right]}
\end{gathered}
$$

where $\hat{D}_{\mathcal{L}}^{-} \equiv \frac{1}{|\overline{\mathcal{L}}|} \sum_{i \in \overline{\mathcal{L}}} K\left(d_{i}\right)$ and $\hat{D}_{\mathcal{L}} \equiv \frac{1}{|\mathcal{L}|} \sum_{i \in \mathcal{L}} K\left(d_{i}\right)$. As $\hat{x}_{l}$ and $\hat{y}_{l}$ are chosen optimally, Eq. (18) reduces to:

$$
\frac{d \hat{g}}{d \lambda}=-\frac{F^{\prime}(g) \hat{D}_{\mathcal{L}}^{-}+H^{\prime}(\hat{g})-1}{H^{\prime \prime}(\hat{g})+F(g)\left[\lambda \hat{D}_{\mathcal{L}}^{-}+\hat{D}_{\mathcal{L}}\right]} .
$$

By the second order condition for a maximum, the denominator is negative. With regard to the sign of the numerator, notice that the first order condition for an interior solution for $\hat{g}$ is given by:

$\lambda\left[F^{\prime}(g) \hat{D}_{\mathcal{L}}^{-}+H^{\prime}(\hat{g})-1\right]+F^{\prime}(g) \hat{D}_{\mathcal{L}}+H^{\prime}(\hat{g})-1=0$.

(a) Consider first the Nimby case with $F^{\prime}(g)<0$. Notice that in equilibrium $\hat{D}_{\mathcal{L}}^{-}$must be larger than $\hat{D}_{\mathcal{L}}$ since lobbies have higher weight in the government's objective function. Thus, it must be:

$F^{\prime}(g) \hat{D}_{\mathcal{L}}^{-}+H^{\prime}(\hat{g})-1<0$,

otherwise Eq. (19) could not be satisfied. Thus, in this case, $d \hat{g} / d \lambda<0$ and $\hat{g}>g^{*}$.

(b) Consider now the Nimby case with $F^{\prime}(g)>0$. By the same line of reasoning as before, it must be true that:

$F^{\prime}(g) \bar{D}_{\mathcal{L}}^{-}+H^{\prime}(\hat{g})-1>0$

so that $d \hat{g} / d \lambda>0$ and $\hat{g}<g^{*}$.

(c) In the Imby case, the proof proceeds exactly as in the Nimby case. Notice that in this case $\hat{D}_{\mathcal{L}}^{-}<\hat{D}_{\mathcal{L}}$.

\section{Appendix E. Proof of Proposition 5}

(i) By definition of $d_{i}^{*}, \hat{d}_{i}, g^{*}$ and $\hat{g}$, we have:

$$
\sum_{i \in \mathcal{L}} v_{i}\left(g^{*}, d_{i}^{*}\right)+\sum_{i \in \overline{\mathcal{L}}} v_{i}\left(g^{*}, d_{i}^{*}\right)>\sum_{i \in \mathcal{L}} v_{i}\left(\hat{g}, \hat{d}_{i}\right)+\sum_{i \in \overline{\mathcal{L}}} v_{i}\left(\hat{g}, \hat{d}_{i}\right)
$$

and

$\sum_{i \in \mathcal{L}} v_{i}\left(g^{*}, d_{i}^{*}\right)+\lambda \sum_{i \in \mathcal{L}} v_{i}\left(g^{*}, d_{i}^{*}\right)<\sum_{i \in \mathcal{L}} v_{i}\left(\hat{g}, \hat{d}_{i}\right)+\lambda \sum_{i \in \mathcal{L}} v_{i}\left(\hat{g}, \hat{d}_{i}\right)$

which can be rewritten as:

$\sum_{i \in \mathcal{L}}\left[v_{i}\left(g^{*}, d_{i}^{*}\right)-v_{i}\left(\hat{g}, \hat{d}_{i}\right)\right]>\sum_{i \in \mathcal{L}}\left[v_{i}\left(\hat{g}, \hat{d}_{i}\right)-v_{i}\left(g^{*}, d_{i}^{*}\right)\right]$

and

$\sum_{i \in \mathcal{L}}\left[v_{i}\left(g^{*}, d_{i}^{*}\right)-v_{i}\left(\hat{g}, \hat{d}_{i}\right)\right]<\lambda \sum_{i \in \mathcal{L}}\left[v_{i}\left(\hat{g}, \hat{d}_{i}\right)-v_{i}\left(g^{*}, d_{i}^{*}\right)\right]$.

Clearly, these two inequalities can be satisfied together if and only if:

$\sum_{i \in \mathcal{L}}\left[v_{i}\left(g^{*}, d_{i}^{*}\right)-v_{i}\left(\hat{g}, \hat{d}_{i}\right)\right]<0$

and

$\sum_{i \in \overline{\mathcal{L}}}\left[v_{i}\left(\hat{g}, \hat{d}_{i}\right)-v_{i}\left(g^{*}, d_{i}^{*}\right)\right]<0$

(ii) Consider a 10 -household $O$-symmetrical structure with $P_{1}$ located at $0, P_{2}$ and $P_{3}$ between 0 and $\pi / 2$ and $P_{4}$ and $P_{5}$ between $\pi / 2$ and $\pi$. Suppose also that the distance between $P_{2}$ and $P_{3}$ is 
arbitrarily small and that the facility is Nimby. If all households lobby, $\hat{L}=O$. Consider now a different structure where $P_{2}$ does not lobby. Then $\hat{L}$ moves closer to $P_{2}$ and inevitably to $P_{3}$. Thus $P_{3}$ will be worse off than in the equilibrium with complete lobbying. Similarly, in the case of an Imby facility, consider $P_{2}$ as the only lobbying household. Then $\hat{L}$ moves closer to $P_{2}$ and inevitably to $P_{3}$. Thus $P_{3}$ will be better off than in the equilibrium with complete lobbying.

\section{References}

Ahlfeldt, G.M., Maennig, W., 2010. Impact of sports arenas on land values: evidence from Berlin. The Annals of Regional Science 44, 205-227.

Bernheim, B.D., Whinston, M.D., 1986. Menu auctions, resource allocation and economic influence. Quarterly Journal of Economics 101, 1-31.

Besfamille, M., Lozachmeur, J.-M., 2010. NIMBY and mechanism design under different constitutional constraints. International Tax and Public Finance 17, 114-132.

Besley, T., Coate, S., 2001. Lobbying and welfare in a representative democracy. The Review of Economic Studies 68, 67-82.

Bhattacharya, U.K., 2011. A multi-objective obnoxious facility location model on a plane. American Journal of Operations Research 1, 39-45.

Facility location, In: Drezner, Z., Hamacher, H. (Eds.), Facility Location. Applications and Theory, 2nd edition. Springer-Verlag.

Feinerman, E., Finkelshtain, I., Kan, I., 2004. On a political solution to the Nimby conflict. The American Economic Review 94, 369-381.

Felli, L., Merlo, A., 2006. Endogenous lobbying. Journal of the European Economic Association $4,180-215$
Fredriksson, P.G., 2000. The siting of hazardous waste facilities in federal systems: the political economy of NIMBY. Environmental and Resource Economics 15, 75-87.

Frey, B.S., Eichenberger, R., Oberhoizer-Gee, F., 1996. The old lady visits your backyard: a tale of morals and markets. Journal of Political Economy 104, 1297-1313.

Grossman, G.M., Helpman, E., 1994. Protection for sale. The American Economic Review 84, 833-850.

Grossman, G.M., Helpman, E., 2001. Special Interest Politics. Princeton University Press. Ingberman, D., 1995. Siting noxious facilities: are markets efficient? Journal of Environmental Economics and Management 29, S-20-S-33.

Kuhn, R., Ballard, K., 1998. Canadian innovations in siting hazardous waste management facilities. Environmental Management 22, 533-545.

Kunreuther, H., Kleindorfer, P.R., 1986. A sealed-bid auction mechanism for siting noxious facilities. The American Economic Review 76, 295-299.

Minehart, D., Neeman, Z., 2002. Effective siting of waste treatment facilities. Journal of Environmental Economics and Management 43, 302-324.

Mitra, D., 1999. Endogenous lobbies formation and endogenous protection: a long-run model of trade determination. The American Economic Review 89, 1116-1134.

Munoz-Perez, J., Saameno-Rodriguez, J.J., 1999. Location of an undesirable facility in polygonal region with forbidden zones. European Journal of Operational Research $114,372-379$.

Ottaviano, G.I.P., Thisse, J.-F., 2002. Integration, agglomeration and the political economics of factor mobility. Journal of Public Economics 83, 429-456.

Snyder, L.V., 2006. Facility location under uncertainty: a review. IIE Transactions 38 (7), 537-554.

Thisse, J.-F., Wildasin, D.E., 1992. Public facility location and urban spatial structure: equilibrium and welfare analysis. Journal of Public Economics 48, 83-118.

Wellisch, D., 1995. Locational choices of firms and decentralized environmental policies with various instruments. Journal of Urban Economics 37, 290-310.

Wolsink, M., 1994. Entanglement of interests and motives: assumptions behind the NIMBY-theory on facility siting. Urban Studies 31, 851-866. 